\title{
Tunable Fano-Resonant Metasurfaces on a Disposable Plastic-Template for Multimodal and Multiplex Biosensing
}

\author{
Rajib Ahmed, Mehmet Ozgun Ozen, Merve Goksin Karaaslan, Cecilia A. Prator, \\ Cassandra Thanh, Shreya Kumar, Leonel Torres, Nikita lyer, Sadie Munter, \\ Sarka Southern, Timothy J. Henrich, Fatih Inci,* and Utkan Demirci*
}

Metasurfaces are engineered nanostructured interfaces that extend the photonic behavior of natural materials, and they spur many breakthroughs in multiple fields, including quantum optics, optoelectronics, and biosensing. Recent advances in metasurface nanofabrication enable precise manipulation of light-matter interactions at subwavelength scales. However, current fabrication methods are costly and time-consuming and have a small active area with low reproducibility due to limitations in lithography, where sensing nanosized rare biotargets requires a wide active surface area for efficient binding and detection. Here, a plastic-templated tunable metasurface with a large active area and periodic metal-dielectric layers to excite plasmonic Fano resonance transitions providing multimodal and multiplex sensing of small biotargets, such as proteins and viruses, is introduced. The tunable Fano resonance feature of the metasurface is enabled via chemical etching steps to manage nanoperiodicity of the plastic template decorated with plasmonic layers and surrounding dielectric medium. This metasurface integrated with microfluidics further enhances the light-matter interactions over a wide sensing area, extending data collection from 3D to 4D by tracking real-time biomolecular binding events. Overall, this work resolves cost- and complexity-related large-scale fabrication challenges and improves multilayer sensitivity of detection in biosensing applications.

Metasurfaces are engineered nanostructured interfaces comprising thin layers of plasmonic or dielectric materials ${ }^{[1]}$ at subwavelength thicknesses that manipulate light by spatially arranged meta-atoms. Those meta-atoms can directly change light properties such as phase, amplitude, and polarization, thus, extending photonic behavior of natural materials. ${ }^{[2]}$ Metasurfaces offer unusual optical phenomena ${ }^{[3]}$ such as optical magnetism, ${ }^{[4]}$ negative reflections, ${ }^{[5]}$ electromagneticinduced transparency, ${ }^{[6]}$ bianisotropy, ${ }^{[7]}$ and Fano resonance. ${ }^{[8,9]}$ In particular, metasurfaces enable optical control by passing through nanostructured arrays to induce a rapid change of phase and light polarization. ${ }^{10]}$ These structures also enable a change of induced light angular moment um, ${ }^{[11]}$ spins and/or quantum states, and provide applications in flat lenses, ${ }^{[12]}$ holograms, ${ }^{[13]}$ invisible clocks, ${ }^{[14]}$ and photonic devices. ${ }^{[15]}$ The light controlling capability of metasurfaces is strongly dependent on engineered nanostructures, especially their shape and material composition. ${ }^{[16]}$ Metasurfaces with different dimensions

Dr. R. Ahmed, Dr. M. O. Ozen, Dr. M. G. Karaaslan, Dr. F. Inci, ${ }^{[+]}$

Prof. U. Demirci

Bio-Acoustic MEMS in Medicine (BAMM) Laboratory

Canary Center at Stanford for Cancer Early Detection

Department of Radiology

Stanford School of Medicine

Palo Alto, CA 94304, USA

E-mail: finci@stanford.edu; utkan@stanford.edu

Dr. C. A. Prator, C. Thanh, S. Kumar, L. Torres, N. Iyer, S. Munter,

Prof. T. J. Henrich

Division of Experimental Medicine

University of California

1001 Potrero Avenue, San Francisco, CA 94110, USA

Dr. S. Southern

School of Medicine

Gaia Medical Institute

505 Coast Boulevard South, La Jolla, CA 92037, USA

The ORCID identification number(s) for the author(s) of this article can be found under https://doi.org/10.1002/adma.201907160.

[+]Present address: UNAM - Institute of Materials Science and Nanotechnology, Bilkent University, Ankara, 06800, Turkey

DOI: 10.1002/adma.201907160 have been fabricated using various nanofabrication methods including nanodisks by template stripping, ${ }^{[17]}$ rod antenna by nanoimprint, ${ }^{[18]}$ retroreflector by electron-beam (e-beam), ${ }^{[19]}$ splitring resonators by nanosphere/ion-beam milling, ${ }^{[20]}$ complex $3 \mathrm{D}$ structures by self-assembly approaches ${ }^{[21]}$ and colloidal synthesis. ${ }^{[22]}$ However, apart from advanced applications, the ability to fabricate cost-effective metasurfaces at a large-scale remains a challenge. A plasmonic metasurface with Fano resonances and asymmetric spectral lines originating from the response of isolated metal atoms, i.e., a unique feature of a quantum system was described recently. ${ }^{[23,24]}$ Metasurfaces are used in a variety of fields, including solar energy harvesting, ${ }^{[25]}$ spectroscopy, ${ }^{[26]}$ sensing, ${ }^{[27]}$ and broadband light manipulation. ${ }^{[28]}$ Importantly, applications of plasmonic metasurface Fano resonances are crucial in biomolecular sensing because they greatly increase the sensitivity. This is achieved by a large spectral shift in resonance frequency in response to interference of two or more molecular oscillations and small perturbations. ${ }^{[23]}$ However, current Fanoresonant metasurfaces ${ }^{[8,23]}$ require complex nanofabrication ${ }^{[6,29]}$ as well as optical sensing techniques ${ }^{[29]}$ that requires expensive 
and bulky equipment (e.g., Fourier transform infrared (FTIR) spectrometers) ${ }^{[26]}$ This critically limits the use of Fano-resonant metasurfaces for point-of-care (POC) biosensing applications.

Detecting and quantifying the interactions of metamaterials with biological entities require a large active area. To address these challenges, others have presented commercial optical disk surfaces as cost-effective bio/chemical sensing platforms. ${ }^{[30,31]}$ Since the biotargets are small and rare in quantity, these platforms require further improvements in resonant characteristics. However, tuning the resonant characteristics of the metamaterial through (i) "etching" a plastic template to control "nanoperiodicity" of the structure, coupled to (ii) precise "deposition" of multilayer plasmonic materials to engineer the plasmonic Fano resonance on a plastic-templated metasurface has not been demonstrated. Here, we show that these tuning processes uniquely enable biosensing applications in detection of nanothick multibiolayers and nanosized biotargets such as virions using off-theshelf, broadly available, inexpensive commercial optical disk surfaces. The Fano-resonant surface integrated with microfluidic realtime measurements did not only help to capture biotargets, but also helped to confine their light-matter interactions within the sensing microenvironment that can be further extended from the 3D collection of the optical information to 4D by tracking the biosensing process over time. Our results indicate that this metasurface offers a portable, cost-effective, disposable plasmonic sensing platform for detection and quantification of multiple molecule layers and virions. Comparing with other techniques, plastictemplated fabrication is easy and enables a large scale, reproducible production of wide-area metasurfaces. We show that plastictemplated metasurface demonstrates tunable Fano resonances due to the coupling/interference between waveguides and surface plasmon polarization (SPP) modes. ${ }^{[32,33]}$ As a result, the metasurface is able to provide maximum sensitivity, $S=377.69 \mathrm{~nm}$ per RIU and figure of merit, FOM $=25$, where sensing performance is comparable with the reported Fano resonance sensor's response in the visible range. ${ }^{[34-37]}$ Our results show real-time, layer-by-layer binding of proteins, antibodies and virus particles achieved by monitoring continuous spectral shifting of asymmetrical Fano-resonant frequency. We demonstrate (1) multiplex read-out from multiple parallel channels or locations on the same channel; (2) specific binding and spectral measurement of antibody-tethering proteins (protein G) and antibodies (anti-gp120) on a layer-by-layer surface chemistry; (3) comprehensive selection of a polyclonal gp120 antibody capable of targeting multiple HIV-1 subtypes (A, B, C, D, E, and BC); (4) multimodal sensing of both multiple types of biomolecules and viruses; (5) detection and quantification of a target protein (recombinant gp120); (6) capture and detection of virus particles (HIV-1, molecular clone YU2), and (7) an inexpensive portable optical platform to detect down to 40 copies $\mu \mathrm{L}^{-1}$ of HIV-1. These results demonstrate that plastic-templated metasurfaces can be used in biosensing applications. Overall, our findings suggest that this metasurface platform can potentially improve clinical diagnostics of diseases such as cancer and HIV/AIDS at resource-limited settings due to its portability and cost-effective features.

The commercial digital versatile disk (DVD) surface design has a built-in, uniform periodic grating with periodicity $\left({ }^{\wedge}\right)$ $\approx 750 \mathrm{~nm}$, width $(W) \approx 450 \mathrm{~nm}$, and height $\approx 23-25 \mathrm{~nm}^{[31]}$ (Figure 1a). To demonstrate the plasmonic response, we have coated the plastic-templated surface grating with multilayers, including titanium (Ti), silver $(\mathrm{Ag})$, and gold $(\mathrm{Au})$ materials through electron beam evaporation technique. We removed unwanted plastic, metals, and photoresist (PR) layers of DVDs to obtain plastic-templated surface grating. Finally, cleaned and chemically etched surface grating was coated with $10 \mathrm{~nm} \mathrm{Ti}$, $30 \mathrm{~nm} \mathrm{Ag}$, and $15 \mathrm{~nm}$ Au layers (optimized based on numerical



b

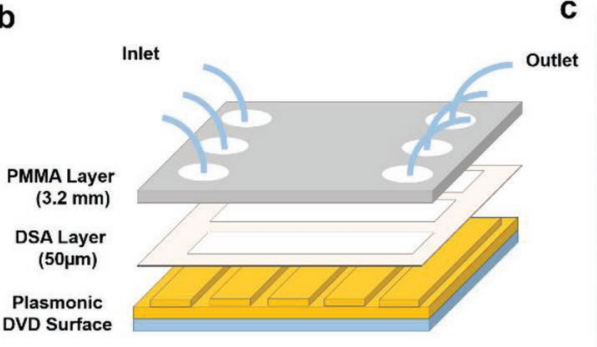

C


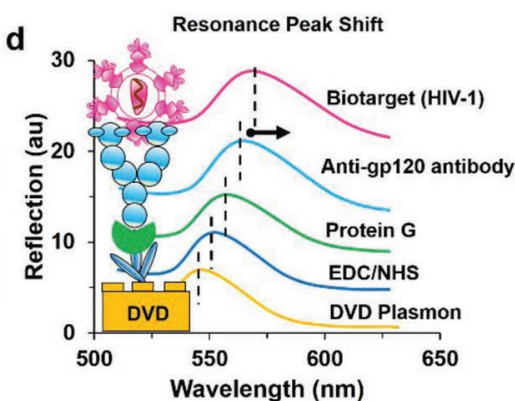

Figure 1. A Fano-resonant plastic-templated metasurface and a portable microfluidic sensor. a) The optical disk surface consists of a dielectric surfacegrating used as a metasurface sensor. The surface was coated with $\mathrm{Ti}(10 \mathrm{~nm}), \mathrm{Ag}(30 \mathrm{~nm})$, and $\mathrm{Au}(15 \mathrm{~nm})$ multilayers to fabricate plastic-templated metasurface. b) Schematic diagram depicts the microfluidic chip consisting of three layers (plasmonic surface, DSA, and PMMA). c) Fabricated metasurface chip is connected with tubing at inlet and outlet ports. d) The plastic-templated metasurface based biosensing measures the layer-by-layer functionalization of biomolecules and target HIV-1 capture. 
modelling). Further details regarding surface preparation, chemical etching (30,60, and $90 \mathrm{~s}$ ), and microfluidic chip assembly steps are illustrated in Figure 2a and the Experimental Section. As a result, the final surface comprises dielectric-metal layers capable of exciting multiple optical modes based on grating (waveguide) dimensions. ${ }^{[38]}$ These surfaces were then integrated with a microfluidic chip for biomolecular detection in real-time (Figure 1b). The assembled microfluidic chip showed a diffractive rainbow pattern that can also be seen by the naked eye (Figure 1c). The device can be disposed of after single-use in clinical or POC settings, where targets can be infectious and biohazardous to avoid contamination; or it can be chemically treated on-chip to completely remove the surface chemistry for re-using the chip in future experimentation in nonbiological analytical chemistry applications. To evaluate plasmonic behavior of the chip, we performed bulk sensing with various concentrations of glycerol solutions. (Figures S1a-c and S2, Supporting Information). The surface sensing with layer-by-layer surface chemistry allowed to monitor the binding of mono- and multilayers of biomolecules and virus particles at the atomic scale (Figure 1d).
The fabrication steps are illustrated in Figure 2a. The fabricated surface showed bright rainbow scattering with the broadband light illumination (Figure $2 \mathrm{~b}(\mathrm{i})$ ). The scanning electron microscopy (SEM) image showed a uniform pattern with a periodicity of $750 \mathrm{~nm}$ (Figure $2 \mathrm{~b}$ (ii)). The atomic force microscopy image confirmed the 3D profile of the surface (Figure 2b(iii)). Uniform grating periodicity on the surface enabled efficient light excitation and coupling of surface plasmon resonance. ${ }^{[39]}$

Near-field electromagnetic excitation of the metasurface resulted in both parallel and antiparallel currents corresponding to the transverse electric (TE) and transverse magnetic (TM) modes. ${ }^{[40]}$ Plastic-templated metasurface waveguides can support multiple TE and TM modes based on waveguide dimension and effective refractive index changes. ${ }^{[41]}$ As refractive index of the surrounding medium increases, effective refractive index value will increase to generate multiple modes and mode-hybridization will occur, accordingly. ${ }^{[42]}$ For plasmonic waveguide-based structures guided modes propagate the light that passes through the metal waveguide, termed as "waveguide (WG) mode." Moreover, SPP modes are generated that propagate along the interface a

i) Top Plastic Removal



ii) PR and Metal Coating Removal

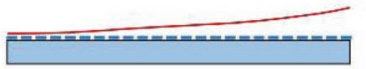

ii) Surface Cleaning with EtOH : MetOH (1:1)

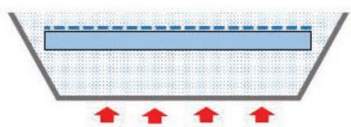

Heat $\left(60^{\circ} \mathrm{C}\right)$

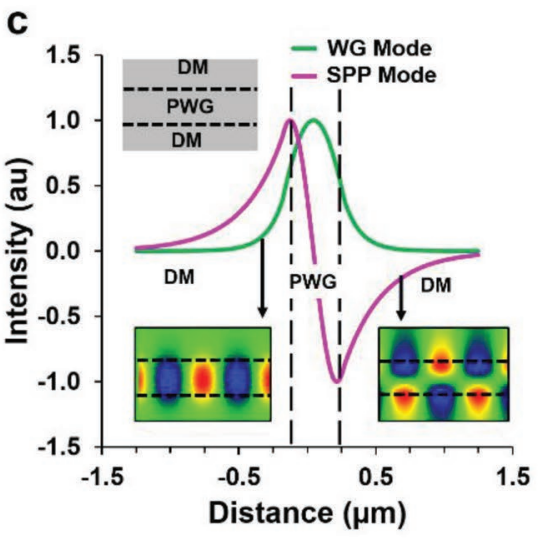

iv) Chemical Etching with Acetone : Isopropanol (1:4)

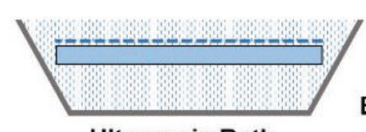

Ultrasonic Bath

v) Plasmonic Metal Coating

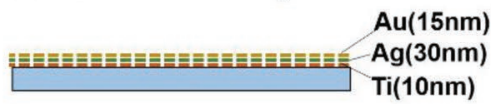

vi) Plasmonic Microfluidic Chip

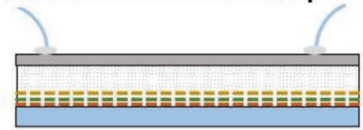

d

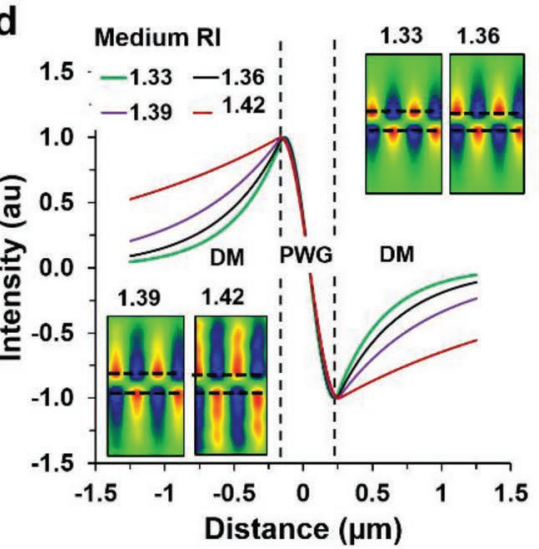

b



iii)
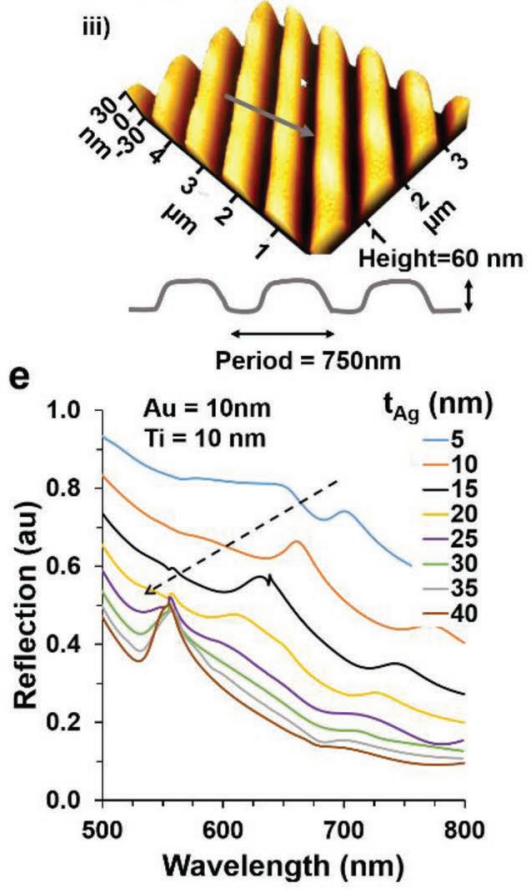

Figure 2. Plastic-templated metasurface fabrication, surface profile, and structure-dependent plasmonic Fano resonance modeling. a) Plastic-templated metasurface fabrication is based on subsequent steps: (i) removal of top plastic, (ii) removal of PR and metal-coating, (iii) surface cleaning, (iv) chemical etching, (v) plasmonic layer coating, and finally (vi) microfluidic chip assembly. b) The fabricated metasurface shows bright rainbow patterns (i), scanning electron microscopy (SEM) image shows uniform surface gratings (ii), and atomic force microscopy (AFM) image depicts 3D profile of coated surface (iii). c) WG and SPP modes are present in the plasmonic waveguide (PWG) grating surface. d) SPP mode response of the metasurface with dielectric medium (DM) RI varying from 1.33 to 1.42 RIU. We have shown plasmonic field distribution at peak resonance frequency, $f_{\text {peak }}=560$ nm in water medium. e) Plasmonic Fano resonance response of metasurface is tuned by changing the thickness of silver (Ag) layer. 
between metal-dielectric sections, defined as "SPP modes" that can be classified as super (bright), and subradiant (dark) modes dependent on coupling strength between incident light from free-space to metasurfaces. ${ }^{[43]}$ Normally, SPP modes can be considered as guided modes that leak-out energy outside the waveguide or not confined to the metasurfaces due to effective RI variation and inherent loss of the metal coating.

Due to the symmetrical metal grating, the subradiant mode will be dominant on the surface. ${ }^{[26]}$ During near-field excitation, WG and SPP modes are excited at the same time. However, based on RI of the dielectric medium, light can be strongly coupled inside the waveguide or leakage to the surrounding medium (Figure 2c). Computational modeling of dielectricmetal interface showed strong dependency on the SPP modes with medium RI variations (Figure $2 \mathrm{~d}$ ). The asymmetric decay of SPP modes increased as we increased the RI of the medium (from 1.33 to 1.42). Therefore, WG modes leaked to the metaldielectric interface and contributed to the interference with the SPP modes. ${ }^{[33]}$ Maximum field-decay occurred with the higher RI of dielectric medium $(n=1.42)$ compared to the lower values $(n=1.33)$. Similarly, the field-decay property was also observed with WG modes due to the variations in medium RI (Figure S1d, Supporting Information). However, the WG mode field decay was lower compared with SPP modes with RI variations.

The plastic-templated metasurface can be considered to function as a plasmonic nanocircuit at optical frequency, where each of multilayer elements with characteristic impedance contributes to the resonance frequency. ${ }^{[4]}$ Accordingly, the plasmonic resonance of surface can be defined with the following equation:

$\lambda_{\text {res }} \approx \frac{p_{0}}{\sqrt{j^{2}+k^{2}}} \sqrt{\frac{\varepsilon_{\mathrm{s}} \varepsilon_{\mathrm{m}}}{\varepsilon_{\mathrm{s}}+\varepsilon_{\mathrm{m}}}}$

where $P_{0}$ is the periodicity of grating surface; $\varepsilon_{\mathrm{s}}$ and $\varepsilon_{\mathrm{m}}$ are permittivity of metal and dielectric medium; and $j$ and $k$ are grating orders. ${ }^{[31]}$ To confirm diffraction patterns, the metal-coated surface was illuminated using a monochromatic light source. The first-order of diffraction pattern was clearly observed (Figures S3 and S4, Supporting Information). When the parameters of grating period $\left(P_{0}=750 \mathrm{~nm}\right)$ and diffraction orders $(i=j=1)$ were kept constant, the resonance frequency was strongly dependent on the material property of the metal and dielectric medium. Moreover, plasmonic response of the plastictemplated metasurface shows asymatical Fano shape. Fano response occurs (based on Ugo Fano in 1961) due to interference between continuum states (background process) and excitation of discrete states (resonant process). ${ }^{[24]}$ Fano resonance of periodic metasurface due to electromagnetic modes, material losses, and their coupling onto the resonance line shape. ${ }^{[4]}$ For our plastic-templated metasurface, asymmetric Fano shape occurs in the reflection spectra due to interference between guided modes from the grating surface (waveguides), which act as a background process and the SPP modes from metaldielectric interfaces that can act as a resonant process. ${ }^{[32,33]}$ The Fano resonance profile of our plastic-templated metasurface can be approximated by following equation: ${ }^{[46]}$

$I_{\text {ref }}(\omega)=\operatorname{Am} \frac{\left[\left(1+\left(\omega-\omega_{c}\right) /(q \Gamma)\right]^{2}\right.}{\left[\left(1+\left(\omega-\omega_{c}\right) / \Gamma\right]^{2}\right.}+\mathrm{BC}$ where $\mathrm{BC}$ is the background term, Am is the amplitude, $\Gamma$ is the resonant bandwidth (BW), and $q$ is the Fano parameters. The value of $q$ is used to define asymmetric property of the Fano profile, which is based on coupling/interference between the background (continuum state) and resonant process (discrete state). Therefore, three special shape of Fano responses are antiresonances $(q=0$, no coupling to the discrete state), asymmetric resonance $(0<q< \pm \infty$, coupling between discrete and continuum states), and Lorentzian shape resonance $(q=\infty$, no coupling to the continuous state). There is no general procedure to design a system with desired $q$ parameter. In addition, $q$ parameter depends on incident light, geometry, and materials properties of a Fano system. ${ }^{[8]}$ Therefore, we have further evaluated the plasmonic response of surfaces by altering material types and coating thicknesses since the metal property (the refractive index or permittivity) of a medium has a strong relationship with its thickness, shape or dimension. ${ }^{[36,47]}$ By employing a finite element method, we computationally assessed the effect of variations in metal layer thicknesses to predict plasmonic responses and the observed plasmonic Fano resonance shift (Figure S5a, Supporting Information). Further, when the thickness of silver layer $\left(t_{\mathrm{Ag}}\right)$ was changed from 5 to $40 \mathrm{~nm}$ whereas the thickness of gold $\left(t_{\mathrm{Au}}=10 \mathrm{~nm}\right)$ and titanium $\left(t_{\mathrm{Ti}}=10 \mathrm{~nm}\right)$ were kept constant, the plasmonic Fano resonance peak had blue-shifted from 707.47 to $558.27 \mathrm{~nm}$, respectively (Figure 2e). Similarly, we also observed blue-shifts in plasmonic Fano resonance dip from 682.22 to $528.73 \mathrm{~nm}$, respectively (Figure $2 \mathrm{e})$. The maximum peak/dip resonance shift $\left(\Delta \lambda_{\text {res }}=\right.$ $41.82 / 44.20 \mathrm{~nm}$ ) was observed when the lower thickness of the silver layer $\left(t_{\mathrm{Ag}}=5 \mathrm{~nm}\right)$ was used. In particular, when the thickness of the silver layer was $\approx 30 \mathrm{~nm}$ and more, the resonance peak and dip remained constant at 558.27 and $530.25 \mathrm{~nm}$, respectively. Therefore, we kept the silver thickness as $30 \mathrm{~nm}$ and titanium thickness as $10 \mathrm{~nm}$ in further simulations. We have also altered the thickness of gold $\left(t_{\mathrm{Au}}\right)$ and titanium $\left(t_{\mathrm{Ti}}\right)$ and measured dip and peak of the plasmonic Fano resonance shifts (Figure S5b,c, Supporting Information). Alteration in titanium thickness did not provide any significant changes in the resonance peak or dip. Then, we have simultaneously altered the thickness of titanium, silver, and gold, and observed similar blue-shift trends as found in the silver thickness variations (Figure S5d, Supporting Information). Therefore, the variation of $t_{\mathrm{Ag}}$ had the dominant effect in the maximum resonance wavelength shifts compared to other metal layers with minimum thickness variations $(5 \mathrm{~nm})$. As earlier reported, ${ }^{[48]}$ the plasmonic response of $\mathrm{Ag}$ nanostructure is stronger than that of $\mathrm{Au}, \mathrm{Ti}$, and other plasmonic materials. Moreover, we compared computational simulations with experimental data and observed the same plasmonic responses for the thickness variations of metal layers (Figure S6a,b, Supporting Information). Moreover, asymatric Fano response tunning due to $q$-value variation through periodic metal surfaces structural parameters (width, height, and thickness) variation are also reported previously. ${ }^{[49]}$

To observe the plasmonic Fano response of the surface with RI variations of dielectric medium, a hand-held, portable (weight $<0.5 \mathrm{lb}$ ) sensing platform was designed and assembled as shown in Figure 3a,b. Briefly, the broadband light is emitted from the source, passes through a series of optical components 
a
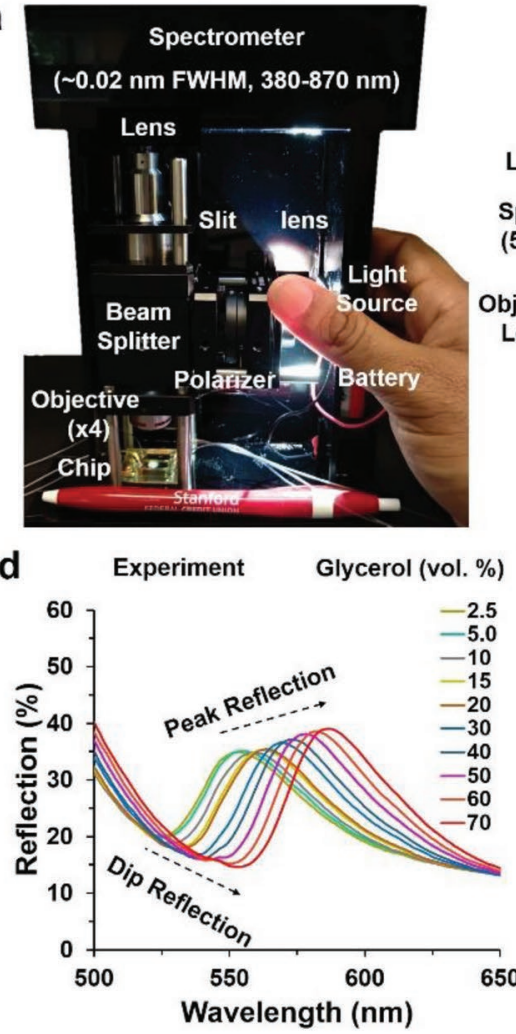

b

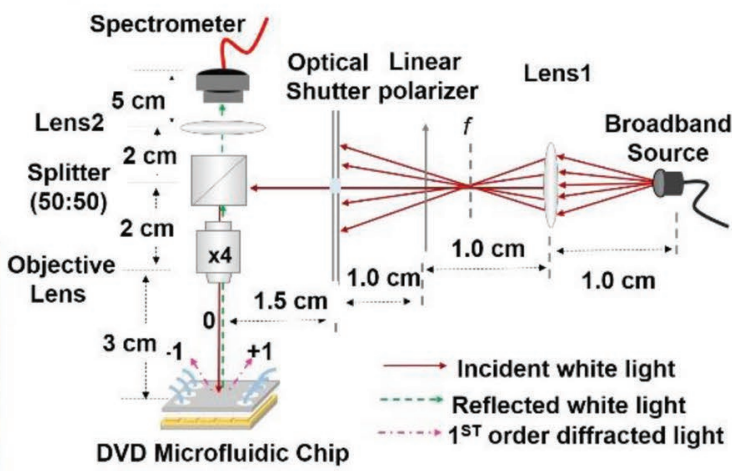

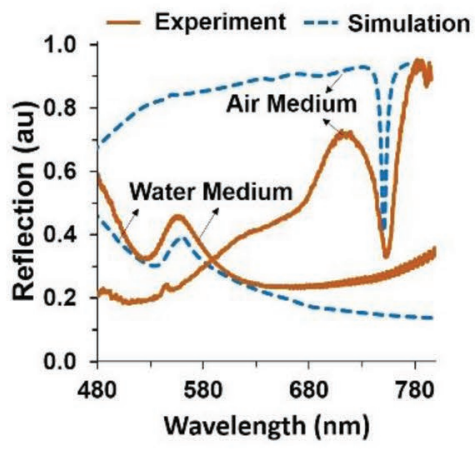

e



f

Figure 3. Bulk Rl-sensing on a portable setup and computational modeling. a) Portable setup measures the plasmonic Fano response of plastictemplated metasurface. b) Conceptual block-diagrams of the optical setup and ray-tracing of the light-transmission and reflection from the metasurface. c) Computational and experiment results demonstrating plasmonic Fano resonance tuning from the metasurfaces with medium (air and water) variations. d) Plasmonic Fano resonance is tuned using a range of glycerol dilutions in water (2.5-70\% v/v). e) Plasmonic Fano resonance wavelength shifts as a function of RI variation. Dotted lines indicate the linearity response during simulation and experiment. f) Real-time dip and peak plasmonic response to the variation in glycerol concentration.

and illuminates the microfluidic-integrated chip. The reflected light is measured through a customized spectrometer (see the Supporting Information). During the system characterization, the plasmonic Fano resonance responses of the plastic surface were measured and acquired using the normal whitelight illumination (Figure 3c). A dip resonance reflection $\left(\lambda_{0}\right)$ was observed at $750 \mathrm{~nm}$ with $15 \mathrm{~nm}$ of spectral line-width or full width at half maximum (FWHM) in air medium. The resonance spectra showed good agreement with computational modeling, and the $3 \mathrm{~dB}$ bandwidth decreased to $5.24 \mathrm{~nm}$. Overall reflection spectra increased by $\approx 10 \%$ due to higher light absorption during experiments. To prove Fano response of the plastic-templated metasurface, we have fitted reflection spectral line with Fano equation and calculated available parameters following Equation (2) (Figure S7, Supporting Information). For air $(n=1.0)$ medium fitting parameter [BC, $\left.\omega_{\mathrm{c},} \mathrm{Am}, \mathrm{BW}, q\right]$ are $[0.92 \pm 003,758.49,-0.479 \pm 0.0047,11.20 \pm 0.215,-2.42 \pm 03]$. Similarly, for water medium, fitting parameter $\left[\mathrm{BC}, \omega_{\mathrm{c}} \mathrm{Am}, \mathrm{BW}\right.$, $q]$ are $[0.102 \pm 001,547.94 \pm 0.35,0.064 \pm 0.001,12.85 \pm 0.79$, 10]. Furthermore, medium-dependent plasmonic Fano resonance tuning response was observed due to the coupling/ interference between the WG and SPP modes. Experimental data and computation modeling of the associated asymmetrical plasmonic Fano resonance response had a similar peak and dip resonance at $\approx 560$ and $525 \mathrm{~nm}$, respectively (Figure $3 \mathrm{c}$ ). However, there is a big different at shorter wavelengths for the reflection in air medium between simulation and experiments conditions. This may be due to light absorption at shorter wavelengths and unable to provide exact experiment environments during modeling.

Optical sensing performance is generally characterized using both spectral shifts $(\Delta \lambda)$ and spectral line-width (FWHM) due to the RI variations $(\Delta n) \cdot{ }^{[39]}$ This is related to the sensor sensitivity $(S=\Delta \lambda / \Delta n)^{[31,34]}$ and figure of merit $(\mathrm{FOM}=\mathrm{S} / \mathrm{FWHM}) \cdot{ }^{\left[{ }^{[3,50]}\right.}$ To observe bulk sensitivity, we have also varied the concentration of glycerol (from $2.5 \%$ to $70 \%, \mathrm{v} / \mathrm{v}$ ), corresponding to the RI variations from 1.336 to 1.428 RIU (a.u.) (Figure 3d,e; Figure S1c,d, Supporting Information). Due to the changes in RI, asymmetric Fano resonance caused a peak red-shift from 553.44 to $585.85 \mathrm{~nm}$, as well as in the dip from 524.32 to $553.22 \mathrm{~nm}$. Similarly, Fano resonance shifts with RI variations were also observed at transmission-mode due to the semitransparent property of fabricated surface (Figure S6c,d, Supporting Information). A linear fit of the resonance wavelength shifting for RI variations demonstrated the sensor sensitivity as $377.69 \mathrm{~nm}$ per RIU and FOM as 25 based on $\Delta \lambda=9.82 \mathrm{~nm}, \Delta n=0.026$ RIU, and FWHM $=15 \mathrm{~nm}$ values, respectively. Computational modeling confirmed the wavelength shifts of the asymmetric 
Fano resonance with different glycerol solutions and correlated with experimental data (Figures S1e and S3e, Supporting Information).

Additionally, we evaluated the effect of etching process (1:4 mixture of acetone and isopropyl alcohol, with different etching periods: 30,60 , and $90 \mathrm{~s}$ ) on the plasmonic Fano resonance response of plastic-templated metasurfaces. Bulk sensing response was measured in real-time with respect to the peak and dip of the Fano resonance with $60 \mathrm{~s}$ etching process (Figure 3f). Both peak and dip resonances were correlated with RI variations in 2.5 to $70 \%$ glycerol solutions. As a result, the peak of Fano resonance provided higher wavelength shifting compared to the dip. Based on the glycerol concentration experiments, we found that $60 \mathrm{~s}$ etched surfaces provided higher wavelength shifts than those of $30 \mathrm{~s}$ and $90 \mathrm{~s}$ etching processes (Figures S6d and S8a,b, Supporting Information). Our sensor performance ( $S$ and FOM) was comparable with the other reported localized surface plasmon resonance platforms in the visible range. ${ }^{[34-37]}$

As Fano resonance is based on the interference of two or more oscillations, a small perturbation can produce a large spectral shift in the resonance frequency. ${ }^{[23,51]}$ To observe this effect, we applied layer-by-layer surface chemistry on the plastic-templated metasurface and monitored real-time biomolecular binding. Chemical steps to capture mono- and multi-biomolecular layers are summarized using a conceptual block diagram in Figure 4a. Briefly, our goal was to optimize the chemical steps to effectively capture recombinant gp120 HIV protein (rec-gp120). Gp120 is an HIV envelope glycoprotein located on the surface of HIV-1 particles. ${ }^{[52]}$ The chemistry steps include $N$-(3-dimethylaminopropyl)- $N$-ethylcarbodiimide hydrochloride (EDC)/ $N$-hydroxysuccinimide (NHS), protein G, anti-gp120 HIV antibody, and rec-gp120 HIV protein. These steps were evaluated on plastic metasurfaces etched for 30, 60 , or $90 \mathrm{~s}$ (Figure 3b). The chemistry details are provided in Table S1 of the Supporting Information. Results showed that the $60 \mathrm{~s}$ etching period provided the maximum cumulative wavelength shift of $0.16,0.36,1.36$, and $1.955 \mathrm{~nm}$ for EDC/NHS, protein G, anti-gp120, and rec-gp120, respectively (Figure 3c). As the complexity of the biomolecule layer increased, the resonance wavelength shifted to the higher values. The dependence

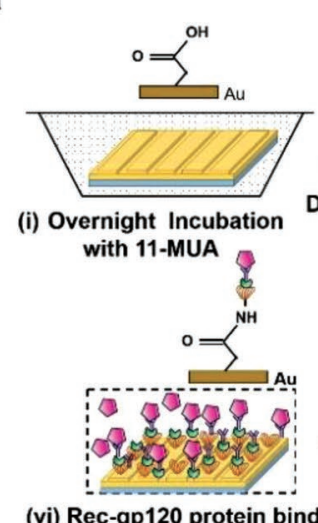

(vi) Rec-gp120 protein binding



(ii) Microfluidic chip assemble

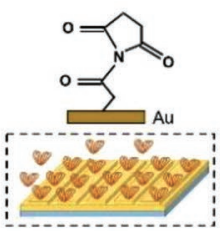

(iii) EDC/NHS binding
Dry Surface
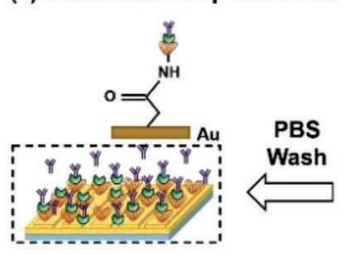

(v) Anti-gp120 antibody binding



b



$\square$ EDC/NHS $\square$ Protein G $\square$ Anti-gp120 $\square$ Rec-gp120
C

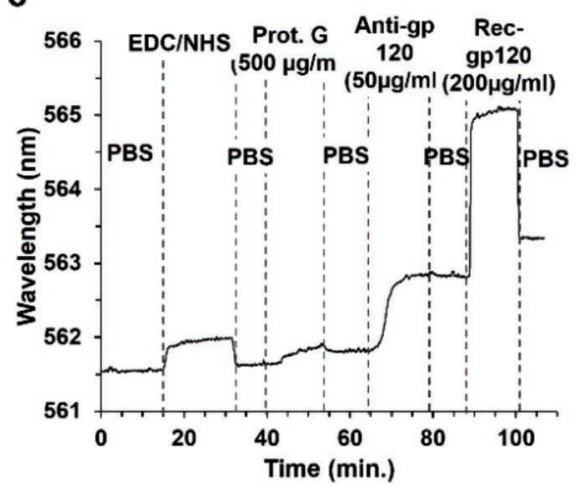

d

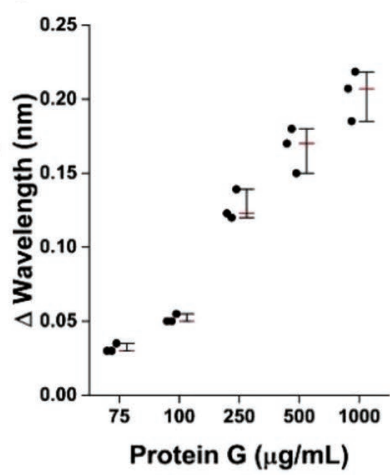

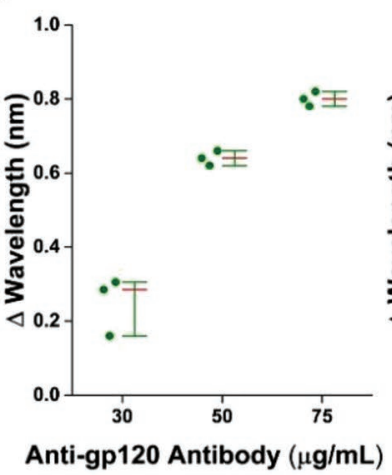

f

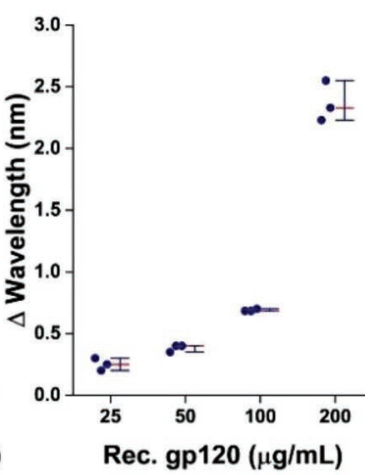

Figure 4. Surface sensitivity of plastic-templated metasurface for layer-by-layer modifications. a) Summary of surface chemistry steps. b) Resonance peak wavelength shifts demonstrated on surfaces etched for 30,60, and $90 \mathrm{~s}$. c) Layer-by-layer binding response of biomolecules on the $60 \mathrm{~s}$ etched surface is monitored in real-time. $d-f$ ) Resonance wavelength shifts as a function various concentrations of protein $\mathrm{G}$, anti-gp 120 antibody, and recgp120 protein are represented via Box-Whisker plots. I-shaped box indicates 25th and 75th, red-line median, and whiskers the 95th and 5th percentiles. Dots represent data points for each concentration. Nonparametric Kruskal-Wallis analysis followed by Dunn's multiple comparison test was performed to evaluate the collected data for each concentration at protein G, anti-gp120 antibody, and rec. gp120 protein experiments. The minimum, first quartile, median, third quartile, and maximum of each concentration were presented in Table S1 of the Supporting Information. In protein G experiment, statistical difference was observed between 75 and $1000 \mu \mathrm{g} \mathrm{mL}^{-1}$ concentrations $(n=3, p<0.05)$. In anti-gp120 antibody experiments, statistical difference was observed between 30 and $75 \mu \mathrm{g} \mathrm{mL}^{-1}$ concentrations $(n=3, p<0.05)$. In rec-gp120 protein experiments, statistical difference was observed between 25 and $200 \mu \mathrm{g} \mathrm{mL}^{-1}$ concentrations $(n=3, p<0.05)$. 
of the resonance wavelength shift $(\Delta \lambda)$ on the molecular layer binding thickness $(d)$ was related to the following equation:

$\Delta \lambda=m\left(n_{\text {layer }}-n_{\text {medium }}\right)\left(1-e^{-2 d / l}\right)$

where $m$ is the sensitivity factor (in nm per RIU); $n_{\text {layer }}$ and $n_{\text {medium }}$ are the refractive indices of the molecule layer (RIU) and dielectric medium, $d$ is the thickness of molecule layer $(\mathrm{nm})$, and $l$ is electromagnetic decay length $(\mathrm{nm}) \cdot{ }^{[35]}$ In our experiment, $m=377.69 \mathrm{~nm}$ per RIU, $n_{\text {layer }}(\approx 1.48),{ }^{[53]}$ having higher value compared to the $n_{\text {medium }}$ (1.33). Therefore, layerby-layer binding of these biomolecules resulted in red-shifts (higher wavelength values). The binding responses of metasurfaces etched for 30 and 90 s periods are shown in Figure S9 of the Supporting Information. To optimize the concentration of protein $\mathrm{G}$, we evaluated a range of concentrations from 75 to $1000 \mu \mathrm{g} \mathrm{mL} \mathrm{m}^{-1}$ and observed cumulative resonance wavelength shifts from $0.032 \pm 0.003$ to $0.204 \pm 0.017 \mathrm{~nm}$ (Figure $4 \mathrm{~d}$; Figure S10, Supporting Information). The lowest concentration of protein $\mathrm{G}\left(50 \mu \mathrm{g} \mathrm{mL} \mathrm{m}^{-1}\right)$ did not provide any signal (data not presented). Protein $G$ data are represented with I-shaped Box-Whisker plots. Through nonparametric Kruskal-Wallis analysis followed by Dunn's multiple comparison test, we observed a statistically difference between 75 and $1000 \mu \mathrm{g} \mathrm{mL}$ concentrations $(n=3, p<0.05)$. Since there was no statistical difference between 500 and $1000 \mu \mathrm{g} \mathrm{mL}^{-1}(n=3, p>0.05)$, we selected the protein $\mathrm{G}$ concentration of $500 \mu \mathrm{g} \mathrm{mL} \mathrm{m}^{-1}$ for further experiments.

On the HIV-1 virus surface, there are several viral proteins that can serve as targets in HIV-1 capture and detection. As an envelope protein, gp120 has discontinuous conserved epitopes (V1-V5 loops) assisting the virus entry to $\mathrm{CD}^{+} \mathrm{T}$ lymphocytes. ${ }^{[54]}$ Therefore, we used this protein as a target protein on the virus surface, and tested the binding of anti-gp120 antibodies to recombinant gp120 proteins. A panel of ten gp120 recombinant proteins representing a broad range of HIV subtypes (A, B, C, D, E, and RC) was tested. Recombinant gp120 proteins were obtained through the National Institutes of Health (NIH), AIDS Research and Reference Reagent Program and also from commercial sources. This panel of recombinant gp120 proteins was applied to authenticate reactivity of antigp120 antibodies using Western Blot analysis (Figure S11a and Table S2, Supporting Information). Twenty anti-gp120 monoclonal and polyclonal antibodies were tested for cross reactivity with multiple HIV subtypes. Two best antibodies were selected: a mouse monoclonal antibody (\#2343 ID6 clone, NIH AIDS Reagent Program) and a rabbit polyclonal antibody (\#11233RP02, Sino Biological) (Figure S11a and Table S2 of the Supporting Information). The polyclonal anti-gp120 antibody was strongly reactive with all 10 gp120 recombinant proteins in the panel (Figure S13a and Table S2 of the Supporting Information). Therefore, the polyclonal antibody was selected for further experiments.

Multiple concentrations of the polyclonal anti-gp120 antibody were evaluated ( 15 to $75 \mu \mathrm{g} \mathrm{mL}{ }^{-1}$ ) on the plastic metasurface. The recorded shifts ranged from $0.25 \pm 0.08$ to $0.8 \pm 0.02 \mathrm{~nm}$ (Figure 4e; Figure S11, Supporting Information). No signal change was detected at the antibody concentration of $15 \mu \mathrm{g} \mathrm{mL} \mathrm{m}^{-1}$ (data not presented). Anti-gp120 antibody data are demonstrated with I-shaped Box-Whisker plots. Through nonparametric Kruskal-Wallis analysis followed by Dunn's multiple comparison test, we observed a statistical difference between 30 and $75 \mu \mathrm{g} \mathrm{mL} \mathrm{m}^{-1}$ concentrations $(n=3, p<$ $0.05)$. Since there was no statistical difference between 50 and $75 \mu \mathrm{g} \mathrm{mL} \mathrm{m}^{-1}(n=3, p>0.05)$, we selected the anti-gp120 antibody concentration of $50 \mu \mathrm{g} \mathrm{mL} \mathrm{m}^{-1}$ for further experiments. The concentrations of rec. gp120 protein ranging from 25 to $200 \mu \mathrm{g} \mathrm{mL} \mathrm{m}^{-1}$ were tested (Figure 4f; Figure S12, Supporting Information). Similarly, rec-gp120 protein data are demonstrated with I-shaped Box-Whisker plots. Through nonparametric Kruskal-Wallis analysis followed by Dunn's multiple comparison test, we observed a statistically difference between 25 and $200 \mu \mathrm{g} \mathrm{mL}^{-1}$ concentrations $(n=3, p<0.05)$. In summary, our plastic-templated metasurface was able to sense multiple biomolecular layers and capture rec-gp120 proteins efficiently based on the capability of the higher order plasmonic modes to cover larger distance into the dielectric medium and efficiently interact with bioanalyte layers .

To capture HIV-1 particles, we modified plastic metasurfaces using the optimized concentrations of 11-mercaptoundecanoic acid (11-MUA), EDC/NHS, protein G, and the polyclonal antigp120 antibody. To model a viral load assay, we diluted the stock HIV-1 YU2 solution using phosphate-buffered saline (PBS). The stock concentration of HIV-1 YU2 was 400 virus copies per $\mu \mathrm{L}$ and the corresponding concentration of the HIV p24 gag protein was $20 \mathrm{pg} \mu \mathrm{L}^{-1}$. We decided to continue using the polyclonal anti-gp120 antibody to make the system versatile to multiple HIV-1 subtypes. We used the plastic-templated surfaces to capture pseudo HIV particles and also HIV virus particles (Figure S13, Supporting Information). We evaluated the effect of surface chemistry on HIV-1 detection, and designed three experimental sets: (1) surface chemistry (no incubation and real-time measurement) and HIV detection (no incubation and real-time measurement); (2) surface chemistry (no incubation and real-time measurement) and HIV detection (with a $1 \mathrm{~h}$ incubation and real-time measurement); and (3) surface chemistry (with incubation) and real-time HIV detection (with a $1 \mathrm{~h}$ incubation in real-time). For HIV sampling, we introduced HIV solution for an hour via a syringe pump with a flow rate of $5 \mu \mathrm{L} \mathrm{min}{ }^{-1}$. The details in surface chemistry and HIV sampling are described in Table S3 of the Supporting Information. In the experimental set-1, HIV detection provided $0.207 \pm 0.133 \mathrm{~nm}$ shift without any incubation steps at both surface chemistry and HIV sampling (Figure S13c, Supporting Information). In the experimental set-2, we observed $0.26 \pm 0.008 \mathrm{~nm}$ shift when we incubated HIV sample for an hour after we introduced the sample to the chip (Figure 5a). In the experimental set-3, incubation steps were included on both the surface chemistry and the HIV capture where we incubated the same HIV sample for an hour after introducing it to the plasmonic chip (Figure 5b). The set-3 provided a strongly increased wavelength shift $(0.92 \pm 0.06 \mathrm{~nm})$ indicating that the incubation step in the chemistry enhanced the signal and provided a wider detection range compared to sets 1 and 2 (Figure 5c). Further, virus incubation on the active surface area of the metasurface allowed the viruses to interact longer with the surface, hence enabling more interactions between the target and the surface for a better detection protocol that is reflected as a higher shift in the resonance and 
a

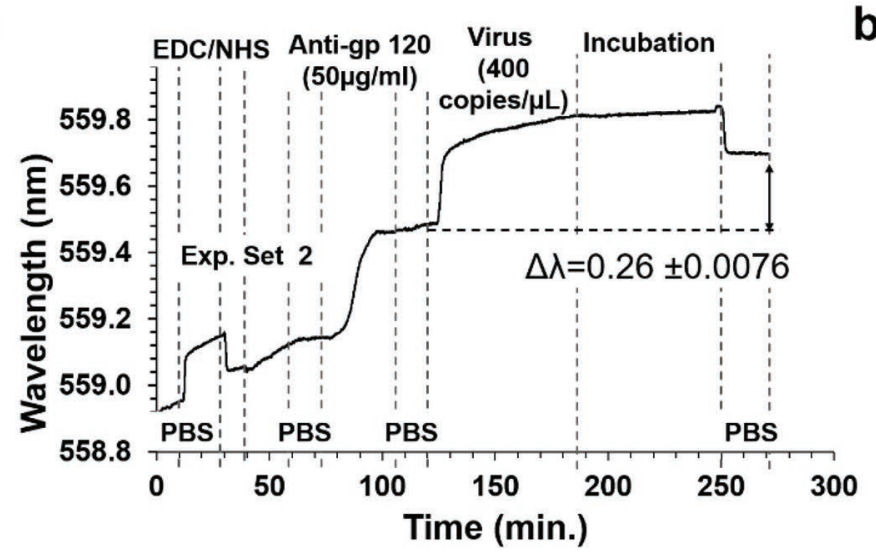

b



e

C



d


Figure 5. Detection and capture of HIV-1 particles. a,b). Real-time detection of HIV-1 YU2 molecular clone is demonstrated for the experimental set-2 and 3. c) Different experimental sets evaluated the effect of extended incubation time on sensing performance. d) Cumulative resonance wavelength shifting is demonstrated usingdifferent HIV-1 concentrationse SEM images of captured HIV-1 particles on both the edge (i) and top (ii) layer of grating surface due to SPP and waveguides modes.

more efficient capture of HIV-1 particles. Dose-response was tested by measuring cumulative binding on the nanoplasmonic surface using three dilutions of the HIV-1 virus YU2 stock in PBS $\left(40,80\right.$, and 200 copies $\mu \mathrm{L}^{-1}$. All HIV dilutions provided red-shifts in the peak wavelength (Figure $5 \mathrm{~d}$ ). The HIV virus was captured at different locations on the chip (both the top and edge side of the metasurface grating) demonstrating that the entire surface was efficiently functionalized with the anti-gp120 antibody (Figure 5e). This indicated that both waveguide and plasmonic modes may contribute to surface plasmon Fano resonance and enable efficient biosensing. Together, these results suggest that the metasurface chip we present here has potential for a high impact on HIV viral load testing.

In summary, we introduce plastic-templated metasurfaces with a large active area, having periodic metal-dielectric layers to excite plasmonic Fano resonance modes providing multimodal and multiplex sensing. Our approach will be able to resolve cost and complexity related with large-scale metasurface fabrication, and enhance sensitivity of layer-by-layer real-time binding of mono- and multilayer protein, antibody, and virus particles on a cost-effective, disposable, and portable platform. Briefly, we designed and developed a plasmonic metasurface, and demonstrated efficient Fano resonance modes and specific detection of protein G, recombinant gp120 protein and HIV viral particles.
We also showed that biosensing properties of these metasurfaces can be monitored using a simple, cost-effective, and portable platform to quantify binding of specific proteins and virus particles in real-time. The plasmonic Fano resonance mode multiplexing capability demonstrated here has potential to be generally applied to detect other specific proteins, viruses, and cells.

The metasurface demonstrated here is cost-effective and can be mass produced. This metasurface preparation method moves the fabrication process from the traditionally used silicon substrate to plastic, leveraging the nanoperiodicity that has been already perfected in the commercial surface. This innovative change in fabrication approach reduces the manufacturing cost by $\approx 100$ times, enables reproducibility over a large surface area and makes possible to control electromagnetic field at nanoscale regions with a high precision. In conclusion, plastic-templated metasurfaces demonstrated here have potential to offer a wide range of applications, including biochemical sensing, optoelectronics, and optical spectroscopy devices.

\section{Experimental Section}

Preparation of Plastic-Templated Metasurfaces: DVD surface consists of top plastic surface, metal-coating, photoresist layers, surface grating, and polycarbonate-plastic layers. Briefly, metasurfaces were prepared by 
removing the coating on DVD surface, unwanted metal-coating, and PR layers using step-by-step physical and chemical processes. Fabrication steps: (1) top coating layer of DVD was removed using a knife; (2) metal-coating was removed using air-flow; (3) surface was cleaned using a 1:1 mixture of ethanol (EtOH) and methanol (MetOH) at $60^{\circ} \mathrm{C}$ and (4) the surface was etched using a 1:4 mixture of acetone and isopropanol for different etching periods $(30,60$, and $90 \mathrm{~s})$. After the cleaning step, (5) DVD surface grating was coated with $10 \mathrm{~nm}$ titanium (Ti), $30 \mathrm{~nm}$ silver $(\mathrm{Ag})$, and $15 \mathrm{~nm}$ gold $(\mathrm{Au})$ layers through electron beam evaporation technique (AJA international, Inc., MA 02066, USA). For $10 \mathrm{~nm}$ Ti layer, $8.3 \mathrm{kV}$ voltage, $60 \mathrm{~mA}$ current, and $0.5 \mathrm{~A} \mathrm{~s}^{-1}$ deposition rate were used. For $30 \mathrm{~nm}$ Ag layer, $8.3 \mathrm{kV}$ voltage, $96 \mathrm{~mA}$ current, and $1 \mathrm{~A} \mathrm{~s}^{-1}$ deposition rate were used. Similarly, $15 \mathrm{~nm}$ Au coating thickness was obtained from $8.3 \mathrm{kV}, 175 \mathrm{~mA}$ current, and $1 \mathrm{~A} \mathrm{~s}^{-1}$ deposition rate parameters. (6) Finally, the plasmonic surface was further washed before the microfluidic chip assembly using the 1:1 mixture of $\mathrm{EtOH}$ and $\mathrm{MetOH}$.

Sensitivity and Binding Properties: To measure bulk sensitivity and observe the binding response of biomolecules, glycerol diluted was used in deionized (DI) water $(2.5$ to $70.0 \% \mathrm{v} / \mathrm{v})$. The metasurface was functionalized using layer-by-layer surface chemistry. To evaluate functionalized surface sensitivity, the coated metasurface was modified using $10 \times 10^{-3} \mathrm{M}$ of 11-MUA (450561, Sigma-Aldrich, MO, USA) overnight to create carboxyl groups. Afterward, the surface was cleaned using 1:1 mixture of $\mathrm{EtOH}$ and $\mathrm{MetOH}$ and dried using filtered air. Then, the surface was assembled using plastic layers to construct microfluidic chip. The microfluidic chip consisted of two layers: (1) a poly(methyl methacrylate) (PMMA, thickness: $3.2 \mathrm{~mm}$, MacMaster Carr, Elmhurst, IL, USA) as the top layer with inlet and oulet ports. (2) A double-sided adhesive (DSA) layer (thickness: $50 \mu \mathrm{m}$, iTapestore, Scotch Plains, NJ, USA) to create microchannels and assemble the chip with surface. The PMMA and DSA surfaces were cut using a $\mathrm{CO}_{2}$ laser cutter (VL-200, VersaLaser, Arizona, USA).

HIV gp120 Protein and Virus Capture: Multilayer immunocapture method was used to capture gp120 HIV protein and HIV viral particles on the metasurfaces in three steps. (1) Protein G layer: After MUA modification, a 1:1 mixture of $100 \times 10^{-3}$ M EDC (03450 Fluka - Sigma-Aldrich, MO, USA) and $100 \times 10^{-3}$ M NHS (130672 AldrichSigma Aldrich, MO, USA) was flowed through the channel to create succinamide groups. The surfaces were then washed with $100 \mu \mathrm{L}$ PBS flow via inlet ports. The succinamide groups reacted with amine groups in protein G (\#21193, Thermo Fisher Scientific, MO, USA). This step was followed by a $100 \mu \mathrm{L}$ PBS wash. (2) Anti-gp 120 antibody layer: $100 \mu \mathrm{L}$ of anti-gp120 (immunoaffinity purified rabbit polyclonal $\mathrm{IgG}, 1 \mathrm{mg} \mathrm{mL} \mathrm{m}^{-1}$, \#11233-RP02, Sino Biological, PA, USA) was captured on the protein $G$ layer and the surface was washed using $100 \mu$ L PBS. (3) HIV capture: the functionalized metasurface was used to capture recombinant gp120 proteins and HIV-1 particles. After the capture events, the surfaces were washed with $100 \mu \mathrm{L}$ of PBS. All the steps were monitored in real-time. Recombinant gp120 proteins representing HIV subtypes A, B, C, D, $\mathrm{E}$, and RC were obtained from the NIH AIDS Research and Reference Reagent Program, Sino Biological Inc. and Immune Technology Corp. (New York, NY). To model a viral load assay, a stock solution of HIV-1 YU2 molecular clone (400 HIV-1 virus copies per $\mu \mathrm{L}$ based on $20 \mathrm{pg}$ of HIV Gag pP24 protein per $\mu \mathrm{L}$ as determined via ELISA, see Virus Production and Quantification section below) was used. The stock solution of HIV-1 YU2 was provided by Dr. Timothy Henrich.

Portable Optical Setup: A portable, customized optical setup was developed to measure the plasmonic responses of the metasurface gratings. Briefly, the portable setup consists of light-emitting diode (LED), AA battery $(3.7 \mathrm{~V})$, convex lens, optical linear polarizer, slit ( $\varnothing$ 0.9-12 mm), beam splitter (50:50), objective lens $(\times 4)$, and a customized spectrometer (Ocean optics, HR4000+ (custom), Resolution: $\approx 0.02 \mathrm{~nm}$ FWHM, detection range: 380-870). Broadband light from an LED source was focused through an input convex lens that passed through a linear polarizer, slit $(50 \mu \mathrm{m})$, a beam splitter (50:50), and finally illuminated to the microfluidic metasurface through an objective $(\times 4)$. The light reflected from the metasurface chip returns to the same objective by passing through the beam splitter and output convex lens. Finally, the response is measured in real-time by using a portable spectrometer. Analyte was flowed through the channel using a syringe pump setup (withdraw mode) to change the refractive index (RI) of the dielectric medium. The resultant Fano resonance wavelength shifting was measured through the spectrometer using MATLAB interfaced omindrive software. Real-time plasmonic response of the metasurface sensor was measured through the peak-wavelength shifts versus analyte flow time (in second).

Simulations: The light reflection guiding property from the plastictemplated metasurface was modeled using a finite element-based computational software (COMSOL Multiphysics, CST Studio Suite). The model is based on the 3D geometry of grating structure (width = $450 \mathrm{~nm}$, height $=25 \mathrm{n}$, and periods $=730 \mathrm{~nm}$ ). Taking advantage of planar symmetry in the $x-y$ plane and periodic grating structures, a single unit cell, with periodic boundary conditions, an input port for light excitation, and an output port to measure the light was modeled. Similar, single unit cell simulation of periodic metasurface was also previously reported in the literature. ${ }^{[5]}$ The grating structure was made using polycarbonate $(n=1.58)$ coated with titanium $(\mathrm{Ti})$, silver $(\mathrm{Ag})$, and gold $(\mathrm{Au})$ materials. A broadband light source illuminated the unit grating surface from the top. Light reflection from the grating surface with medium RI variation (from 1.33 to 1.42) was collected from the entrance of the incident beam.

Virus Production and Quantification: HIV particles were prepared from HIV-1 YU2, a CCR5-using molecular clone that productively infects primary lymphocytes, monocytes, and macrophages. pYU2 plasmid was obtained from Dr. Beatrice Hahn and Dr. George Shaw (NIH AIDS Reagent Program, cat\# 1350). Briefly, 293T cells were transfected with pYU2 using the X-tremeGENE 9 DNA transfection reagent following the manufacturer protocol (Millipore Sigma). Cells were incubated at $37^{\circ} \mathrm{C}$ for $48-72 \mathrm{~h}$ in DMEM plus $10 \%$ fetal bovine serum, penicillin, and streptomycin. Supernatants were removed, clarified by passage through a $0.45 \times 10^{-6} \mathrm{M}$ mesh filter, aliquoted, and frozen at $-80^{\circ} \mathrm{C}$ for future use. Viral stocks were quantified using real-time PCR amplification of HIV-1 RNA as previously reported ${ }^{[2]}$ and HIV-1 P24 gag protein was detected using the Alliance HIV-1 p24 ELISA kit (Perkin Elmer) following the manufacturer protocol.

Western Blotting: HIV recombinant proteins were analyzed using $7.5 \%$ Criterion SDS-PAGE gels (Bio-Rad cat\# 5678025) and Trans-Blot Turbo Transfer Packs (Nitrocellulose, Bio-Rad cat\# 170-4159). Criterion gels made possible QC monitoring across the workflow: total protein was UV imaged in the gel and on the nitrocellulose blot to evaluate molecular integrity and transfer efficiency. The gp120 HIV protein was detected using $\mathrm{ECL}$ sandwich immunoassay with the same antibody as used in the metasurface system (rabbit polyclonal anti-gp120 cat\# 11233-RP02 Sino Biological, PA, USA), goat antirabit HRP conjugate (cat\# 111-035045, Jackson ImmunoResearch Laboratories, West Grove, PA, USA), and West Femto Maximum Sensitivity Substrate (Thermo Fisher Scientific, cat \# 34096). The chemiluminescent signal was recorded as a series of exposures (typically $1 \mathrm{~s}-30 \mathrm{~min}$ ) using a digital camera (Chemidoc MP, BioRad).

Statistical Analysis: Statistical assesements were performed using GraphPad Prism software. Details on the statistical analysis are indicated in the text and figure captions.

\section{Supporting Information}

Supporting Information is available from the Wiley Online Library or from the author.

\section{Acknowledgements}

R.A. and M.O.O. contributed equally to this work. This work was supported by the National Institutes of Health (NIH, Nos. R01 DE024971, 
R44 DE021672, and R01 Al120683). M.G.K. was a visiting postdoctoral researcher at Stanford University and was supported by a fellowship from the Scientific and Technological Research Council of Turkey.

\section{Conflict of Interest}

Prof. Utkan Demirci (UD) is a founder of and has an equity interest in: (i) DxNow Inc., a company that is developing microfluidiclVF tools and imaging technologies for point-of-care diagnostic solutions, (ii) Koek Biotech, a company that is developing microfluidic technologies for clinical solutions, (iii) Levitas Inc., a company focusing on developing microfluidic products for sorting rare cells from liquid biopsy in cancer and other diseases, and (iv) Hillel Inc., a company bringing microfluidic cell phone tools to home settings.UD's interests were viewed and managed in accordance with the conflict of interest policies.

\section{Keywords}

biosensing, metasurfaces, microfluidics, point-of-care diagnostics, tunable Fano resonances

Received: October 31, 2019

Revised: December 25, 2019

Published online: March 23, 2020

[1] a) X. Zang, F. Dong, F. Yue, C. Zhang, L. Xu, Z. Song, M. Chen, P. Y. Chen, G. S. Buller, Y. Zhu, Adv. Mater. 2018, 30, 1707499; b) B. Su, Y. Wu, Y. Tang, Y. Chen, W. Cheng, L. Jiang, Adv. Mater. 2013, 25, 3968.

[2] A. A. High, R. C. Devlin, A. Dibos, M. Polking, D. S. Wild, J. Perczel, N. P. de Leon, M. D. Lukin, H. Park, Nature 2015, 522, 192.

[3] A. S. Karimullah, C. Jack, R. Tullius, V. M. Rotello, G. Cooke, N. Gadegaard, L. D. Barron, M. Kadodwala, Adv. Mater. 2015, 27, 5610

[4] a) S. Linden, C. Enkrich, M. Wegener, J. Zhou, T. Koschny, C. M. Soukoulis, Science 2004, 306, 1351; b) T.-J. Yen, W. Padilla, N. Fang, D. Vier, D. Smith, J. Pendry, D. Basov, X. Zhang, Science 2004, 303, 1494.

[5] a) D. R. Smith, W. J. Padilla, D. Vier, S. C. Nemat-Nasser, S. Schultz, Phys. Rev. Lett. 2000, 84, 4184; b) R. A. Shelby, D. R. Smith, S. Schultz, Science 2001, 292, 77.

[6] Y. Yang, I. I. Kravchenko, D. P. Briggs, J. Valentine, Nat. Commun. 2014, 5, 5753

[7] R. Marqués, F. Medina, R. Rafii-El-Idrissi, Phys. Rev. B 2002, 65, 144440.

[8] M. F. Limonov, M. V. Rybin, A. N. Poddubny, Y. S. Kivshar, Nat. Photonics 2017, 11, 543 .

[9] S. Zeng, K. V. Sreekanth, J. Shang, T. Yu, C. K. Chen, F. Yin, D. Baillargeat, P. Coquet, H. P. Ho, A. V. Kabashin, Adv. Mater. 2015, 27, 6163.

[10] A. V. Kildishev, A. Boltasseva, V. M. Shalaev, Science 2013, 339, 1232009.

[11] E. Karimi, S. A. Schulz, I. De Leon, H. Qassim, J. Upham, R. W. Boyd, Light Sci. Appl. 2014, 3, e167.

[12] C. Williams, Y. Montelongo, T. D. Wilkinson, Adv. Opt. Mater. 2017, $5,1700811$.

[13] H. Butt, Y. Montelongo, T. Butler, R. Rajesekharan, Q. Dai, S. G. Shiva-Reddy, T. D. Wilkinson, G. A. Amaratunga, Adv. Mater. 2012, 24, OP331.

[14] X. Ni, Z. J. Wong, M. Mrejen, Y. Wang, X. Zhang, Science 2015, 349, 1310
[15] K. Xiong, G. Emilsson, A. Maziz, X. Yang, L. Shao, E. W. Jager, A. B. Dahlin, Adv. Mater. 2016, 28, 9956.

[16] a) N. Meinzer, W. L. Barnes, I. R. Hooper, Nat. Photonics 2014, 8, 889; b) A. Boltasseva, H. A. Atwater, Science 2011, 331, 290; c) C. Wang, W. Zhang, Z. Zhao, Y. Wang, P. Gao, Y. Luo, X. Luo, Micromachines 2016, 7, 118 .

[17] W. Zhou, T. W. Odom, Nat. Nanotechnol. 2011, 6, 423.

[18] G. Vecchi, V. Giannini, J. G. Rivas, Phys. Rev. Lett. 2009, 102, 146807.

[19] A. Arbabi, E. Arbabi, Y. Horie, S. M. Kamali, A. Faraon, Nat. Photonics 2017, 11, 415

[20] a) M. Decker, N. Feth, C. M. Soukoulis, S. Linden, M. Wegener, Phys. Rev. B 2011, 84, 085416; b) A. E. Nikolaenko, F. De Angelis, S. A. Boden, N. Papasimakis, P. Ashburn, E. Di Fabrizio, N. I. Zheludev, Phys. Rev. Lett. 2010, 104, 153902.

[21] J. Biener, G. W. Nyce, A. M. Hodge, M. M. Biener, A. V. Hamza, S. A. Maier, Adv. Mater. 2008, 20, 1211.

[22] P. Yang, J. Zheng, Y. Xu, Q. Zhang, L. Jiang, Adv. Mater. 2016, 28, 10508.

[23] A. B. Khanikaev, C. Wu, G. Shvets, Nanophotonics 2013, 2, 247.

[24] U. Fano, Phys. Rev. 1961, 124, 1866.

[25] C. Wu, B. NeunerllI, J. John, A. Milder, B. Zollars, S. Savoy, G. Shvets, J. Opt. 2012, 14, 024005.

[26] C. Wu, A. B. Khanikaev, R. Adato, N. Arju, A. A. Yanik, H. Altug, G. Shvets, Nat. Mater. 2012, 11, 69.

[27] T. J. Henrich, E. Hanhauser, Z. Hu, H.-J. Stellbrink, C. Noah, J. N. Martin, S. G. Deeks, D. R. Kuritzkes, F. Pereyra, AIDS 2015, 29, 867.

[28] C. Wu, A. B. Khanikaev, G. Shvets, Phys. Rev. Lett. 2011, 106, 107403.

[29] F. Yesilkoy, E. R. Arvelo, Y. Jahani, M. Liu, A. Tittl, V. Cevher, Y. Kivshar, H. Altug, Nat. Photonics 2019, 13, 390.

[30] a) X. Dou, B. M. Phillips, P.-Y. Chung, P. Jiang, Opt. Lett. 2012, 37, 3681; b) X. Dou, P.-Y. Chung, P. Jiang, J. Dai, Appl. Phys. Lett. 2012 , 100, 041116; c) A. Radu, Y. Y. Ussembayev, M. Jahn, U. Schubert, K. Weber, D. Cialla-May, S. Hoeppener, A. Heisterkamp, J. Popp, RSC Adv. 2016, 6, 44163.

[31] R. Ahmed, M. O. Ozen, F. Inci, M. G. Karaaslan, T. J. Henrich, $\mathrm{U}$. Demirci, presented at Plasmonics in Biology and Medicine XVI, San Francisco, CA, USA 2019.

[32] L. Yang, J. Wang, L.-z. Yang, Z.-D. Hu, X. Wu, G. Zheng, Sci. Rep. 2018, 8, 2560.

[33] S. Hayashi, D. V. Nesterenko, Z. Sekkat, J. Phys. D 2015, 48 325303.

[34] A. I. Kuznetsov, A. B. Evlyukhin, M. R. Gonçalves, C. Reinhardt, A. Koroleva, M. L. Arnedillo, R. Kiyan, O. Marti, B. N. Chichkov, ACS Nano 2011, 5, 4843.

[35] J. N. Anker, W. P. Hall, O. Lyandres, N. C. Shah, J. Zhao, R. P. Van Duyne, Nanoscience and Technology: A Collection of Reviews from Nature Journals, World Scientific, Singapore 2010, p. 308.

[36] H. Chen, X. Kou, Z. Yang, W. Ni, J. Wang, Langmuir 2008, 24, 5233.

[37] A. E. Miroshnichenko, Y. S. Kivshar, Nano Lett. 2012, 12, 6459.

[38] Z. Li, M.-H. Kim, C. Wang, Z. Han, S. Shrestha, A. C. Overvig, M. Lu, A. Stein, A. M. Agarwal, M. Lončar, Nat. Nanotechnol. 2017, 12,675 .

[39] G. Ruffato, G. Zacco, F. Romanato, Plasmonics: Principles and Applications, IntechOpen, Rijeka, Croatia 2012.

[40] F. J. Rodríguez-Fortuño, G. Marino, P. Ginzburg, D. O'Connor, A. Martínez, G. A. Wurtz, A. V. Zayats, Science 2013, 340, 328.

[41] R. Ahmed, A. A. Rifat, A. Sabouri, B. Al-Qattan, K. Essa, H. Butt, Opt. Commun. 2016, 370, 183.

[42] R. F. Oulton, V. J. Sorger, D. Genov, D. Pile, X. Zhang, Nat. Photonics 2008, 2, 496.

[43] S. Zhang, D. A. Genov, Y. Wang, M. Liu, X. Zhang, Phys. Rev. Lett. 2008, 101, 047401.

[44] N. Engheta, Science 2007, 317, 1698. 
[45] B. Gallinet, O. J. Martin, Phys. Rev. B 2011, 83, 235427.

[46] H.-J. Li, L.-L. Wang, X. Zhai, J. Appl. Phys. 2016, 119, 243101.

[47] T. El-Brolossy, T. Abdallah, M. B. Mohamed, S. Abdallah, K. Easawi, S. Negm, H. Talaat, Eur. Phys. J.: Spec. Top. 2008, 153, 361.

[48] G. Albrecht, M. Ubl, S. Kaiser, H. Giessen, M. Hentschel, ACS Photonics 2018, 5, 1058.

[49] K.-L. Lee, J.-B. Huang, J.-W. Chang, S.-H. Wu, P.-K. Wei, Sci. Rep. 2015, 5, 8547.

[50] Y. Yang, I. I. Kravchenko, D. P. Briggs, J. Valentine, Nat. Commun. 2014, 5, 5753.

[51] B. Luk'yanchuk, N. I. Zheludev, S. A. Maier, N. J. Halas, P. Nordlander, H. Giessen, C. T. Chong, Nat. Mater. 2010, 9, 707.

[52] V. Yoon, M. Fridkis-Hareli, S. Munisamy, J. Lee, D. Anastasiades, L. Stevceva, Curr. Med. Chem. 2010, 17, 741.
[53] J. Vörös, Biophys. J. 2004, 87, 553.

[54] a) Y. Li, S. A. Migueles, B. Welcher, K. Svehla, A. Phogat, M. K. Louder, X. Wu, G. M. Shaw, M. Connors, R. T. Wyatt, Nat. Med. 2007, 13, 1032; b) T. Zhou, L. Xu, B. Dey, A. J. Hessell, D. Van Ryk, S.-H. Xiang, X. Yang, M.-Y. Zhang, M. B. Zwick, J. Arthos, Nature 2007, 445, 732; c) X. Wu, Z.-Y. Yang, Y. Li, C.-M. Hogerkorp, W. R. Schief, M. S. Seaman, T. Zhou, S. D. Schmidt, L. Wu, L. Xu, Science 2010, 329, 856; d) R. Wyatt, P. D. Kwong, E. Desjardins, R. W. Sweet, J. Robinson, W. A. Hendrickson, J. G. Sodroski, Nature 1998, 393, 705.

[55] a) C. Hu, X. Wu, R. Tong, L. Wang, Y. Huang, S. Wang, B. Hou, W. Wen, NPG Asia Mater. 2018, 10, 417; b) S. Chen, Z. Li, W. Liu, H. Cheng, J. Tian, Adv. Mater. 2019, 31, 1802458. 